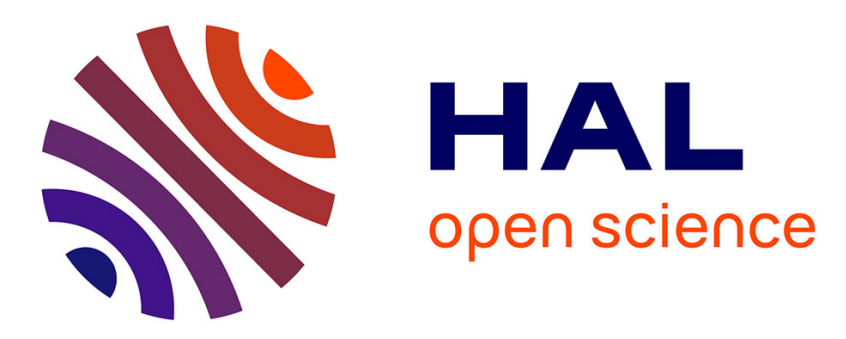

\title{
Preference Aggregation in the Generalised Unavailable Candidate Model
}

Arnaud Grivet Sébert, Nicolas Maudet, Patrice Perny, Paolo Viappiani

\section{To cite this version:}

Arnaud Grivet Sébert, Nicolas Maudet, Patrice Perny, Paolo Viappiani. Preference Aggregation in the Generalised Unavailable Candidate Model. 7th International Conference on Algorithmic Decision Theory, University of Toulouse, Nov 2021, Toulouse, France. pp.35-50, 10.1007/978-3-030-87756-9_3 . hal-03384439

\section{HAL Id: hal-03384439 \\ https://hal.sorbonne-universite.fr/hal-03384439}

Submitted on 18 Oct 2021

HAL is a multi-disciplinary open access archive for the deposit and dissemination of scientific research documents, whether they are published or not. The documents may come from teaching and research institutions in France or abroad, or from public or private research centers.
L'archive ouverte pluridisciplinaire HAL, est destinée au dépôt et à la diffusion de documents scientifiques de niveau recherche, publiés ou non, émanant des établissements d'enseignement et de recherche français ou étrangers, des laboratoires publics ou privés. 


\title{
Preference Aggregation in the Generalised Unavailable Candidate Model
}

\author{
Arnaud Grivet Sébert ${ }^{1}$, Nicolas Maudet ${ }^{2}$, Patrice Perny ${ }^{2}$, and Paolo Viappiani ${ }^{2}$ \\ 1 Université Paris-Saclay, CEA, List, F-91120 Palaiseau, France ${ }^{\star \star}$ \\ arnaud.grivetsebert@gmail.com \\ 2 Sorbonne Université, CNRS, LIP6, F-75005 Paris, France \\ \{nicolas.maudet, patrice.perny, paolo.viappiani\}@lip6.fr
}

\begin{abstract}
While traditional social choice models assume that the set of candidates is known and fixed in advance, recently several researchers $[15,5,2,7,18]$ have proposed to reject this hypothesis. In particular, the unavailable candidate model of $\mathrm{Lu}$ and Boutilier [15] considers voting situations in which some candidates may not be available and focuses on minimising the number of binary disagreements between the voters and the consensus ranking. In this paper, we extend this model and present two new voting rules based on a finer notion of disagreement, called dissatisfaction. The dissatisfaction of a voter is defined as the disutility gap between its preferred available candidate and the candidate elected by the consensus ranking. In the first approach, called ex ante dissatisfaction rule, the disutility is independent of the set of available candidates whereas the second approach, called ex post dissatisfaction rule, assumes that the disutility depends on which candidates are actually available. We provide several results for the two rules. On the one hand, we show that the ex ante rule actually coincides with standard positional scoring rules; therefore, a consensus ranking can be computed in polynomial time. On the other hand, we exhibit strong links between ex post rule and Kemeny rule and we provide a polynomial-time approximation scheme (PTAS) for the ex post problem.
\end{abstract}

Keywords: Computational Social Choice · Preference Aggregation · Unavailable Candidate Model · Polynomial-Time Approximation Scheme.

\section{Introduction}

Traditional social choice theory assumes that the set of candidates is well known before voting takes place. This assumption is not always valid especially in computer science applications (such as recommender systems, decision aid tools, electronic commerce applications) but also in more traditional settings, such as choosing a candidate for a job (a candidate may accept a different job after the hiring committee made its decision). In recent years, several approaches have

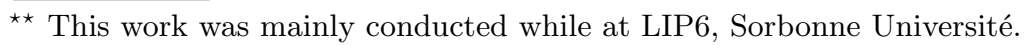


been proposed to address the problem of candidates' unavailability, in particular within computational social choice [6]. Among these approaches, strategic candidatures have been studied extensively $[8,9,13]$. Some works have dealt with the problem of finding robust winners when considering the addition of new candidates [7] or in a context where it is possible to "query" the availability of the candidates [5]. Oren et al. [18] study how many candidates the voters have to rank to ensure the true winner with high probability despite the unavailability hypothesis. Top-k voting may also be a way to deal with unavailability in [17].

An approach of particular interest is the unavailable candidate model (UCM) proposed by $\mathrm{Lu}$ and Boutilier [15]. It assumes that candidates may become unavailable after voters expressed their preferences; therefore there is a need to make decisions in the face of uncertain candidate availability. The optimal rankings are computed by minimisation of the expected number of disagreements over all the possible subsets of available candidates. In [15], the probability distribution on these subsets is supposed to follow a Bernoulli law whereas several other authors $[12,2,11]$ used other distributions. Lu and Boutilier provide a clear decision-theoretic justification for producing a ranking instead of a single winner. Indeed, a ranking serves as a very compact decision policy: the winner is the output ranking's best candidate among the available ones. Yet, the binary disagreement used in $[15,12,2,11]$ relies on strong hypotheses that can be discussed, as acknowledged by the authors: a voter is satisfied only if its favourite available candidate is elected (as in "plurality" rule) and fully unsatisfied otherwise.

We argue that the voter's satisfaction should vary more smoothly and depend on the rank it gives to the candidate declared as winner by the aggregation. In this paper, we extend the UCM by assuming that positions are associated with disutility values and compute dissatisfaction as the disutility gap between the voter's preferred available candidate and the candidate declared as winner. The goal is then to produce a ranking that minimises the expected dissatisfaction under the probability distribution on the subsets of candidates. We observe that there are two opposed ways to measure the satisfaction of the voters, either by considering the ranks of the candidates in the whole preference order of the voter (ex ante approach), or the ranks of the candidates within the subset of available candidates (ex post approach). Hence, we analyse our generalisation of the UCM from these two perspectives; we also show connections to other voting schemes.

We introduce background and notations, and review the UCM in Section 2. In Section 3, we present our framework and introduce the two different models, ex ante and ex post dissatisfactions, that we thoroughly analyse in Sections 4 and 5 respectively. Finally we provide concluding remarks (Section 6).

\section{Background}

Throughout the paper, given a set $\mathscr{E}, \mathscr{P}(\mathscr{E})$ denotes the set of all the subsets (powerset) of $\mathscr{E}$ and $|\mathscr{E}|$ denotes the cardinality of $\mathscr{E}$. We define $\llbracket 1 ; m \rrbracket:=$ $\{1, \ldots, m\}$. We now present our basic assumptions (Section 2.1) and we summarise the UCM (Section 2.2). 


\subsection{Basic assumptions}

We assume that there are $m$ candidates and we call $C$ the set of all the candidates. Given a non-empty subset of candidates $S \subseteq C$, we use $R_{S}$ to denote the set of rankings (permutations) of the candidates of $S$. We use $R$ without subscript to mean $R_{C}$, the set of full rankings involving all candidates in $C$. A ranking can be represented explicitly by the tuple that lists the candidates from the most to the least preferred; for instance, the tuple $(b, c, a)$ denotes the ranking that ranks $b$ in first position, $c$ in second position and $a$ in last position. Given a ranking $r, r_{i}$ denotes the candidate ranked in $i$-th position by $r$. For example, if $r=(b, c, a), r_{1}=b, r_{2}=c$ and $r_{3}=a$.

We suppose that, for every voter $v$, the preferences of $v$ over the candidates can be modelled by a ranking. We will then identify voters with their associated rankings and all the definitions that apply to rankings implicitly apply to voters and vice-versa. Given a ranking $r \in R$, the associated preference order is denoted as $>_{r}$ (and derived orders $\geq_{r},<_{r}$ and $\leq_{r}$ have the obvious meanings). For example, $a$ and $b$ being two candidates, $a>_{r} b$ means that $a$ is better ranked (has a lower rank) than $b$ in $r$.

Given a ranking $r \in R$ and a candidate $a \in C, r(a)$ denotes the position (rank) given to $a$ by $r$. If $S$ is a non-empty subset of $C$, the expression $r_{S}(a)$ denotes the rank of $a$ in the restriction of ranking $r$ that considers only elements of $S$. In other words, $r_{S}(a)=1+\left|\left\{b \in S \mid b>_{r} a\right\}\right|$. In particular, $r_{C}(a)=r(a)$. Using these notations amounts to see $r$ as the bijection that maps a candidate $a \in C$ to its rank $r(a) \in \llbracket 1 ; m \rrbracket$ in the whole set of candidates ${ }^{3}$ and to see $r_{S}$ as the bijection that maps a candidate $a \in S$ to its $\operatorname{rank} r_{S}(a) \in \llbracket 1 ;|S| \rrbracket$ in the subset $S$. Given a non-empty subset $S$ of $C$ and a ranking $r \in R$, $\operatorname{top}_{r}(S)$ is the candidate which is the most preferred by $r$ among the candidates of $S$. In other words, $\operatorname{top}_{r}(S)=r_{S}^{-1}(1)$ if we identify $r_{S}$ with the bijection from $S$ to $\llbracket 1 ;|S| \rrbracket$ as explained above. By convention, $\operatorname{top}_{r}(\emptyset)=a_{\emptyset}$ where $a_{\emptyset}$ is a default alternative when none of the candidates are available (for instance, postpone the election). Another convention is that, if $r$ is a ranking, $r_{\emptyset}\left(a_{\emptyset}\right)=r\left(a_{\emptyset}\right)=1^{4}$.

Under the assumption of anonymity, we will consider voting situations [4] that we here model as multisets of rankings (since the same ranking may occur several times). Considering $n \in \mathbb{N}$ fixed throughout the paper, we use $\mathscr{V}$ to denote the collection of the multisets of $n$ rankings (representing voters).

Given a ranking $r$ and $i \in \llbracket 1 ; m \rrbracket$, we introduce $S_{i}(r)=\left\{a \in C \mid a \leq_{r} r_{i}\right\}=$ $\left\{r_{j} \mid j \in \llbracket i ; m \rrbracket\right\}$, the set of candidates that, in the ranking $r$, are in position $i$ or worse and $\mathscr{S}_{i}(r)=\mathscr{P}\left(S_{i}(r)\right)$ the powerset of $S_{i}(r)$. In addition, $\mathscr{T}_{i}(r)=$ $\left\{S \cup\left\{r_{i}\right\} \mid S \in \mathscr{S}_{i}(r)\right\}=\left\{S \in \mathscr{S}_{i}(r) \mid r_{i} \in S\right\}$ is the collection of all sets of candidates that contain $r_{i}$, and no candidate better than $r_{i}$ in the ranking $r$. Note that $\mathscr{T}_{i}(r)$ is also equal to $\left\{S \subseteq C \mid \operatorname{top}_{r}(S)=r_{i}\right\}$ i.e. the collection of all sets of candidates whose top-element according to $r$ is $r_{i}$.

\footnotetext{
${ }^{3}$ With this point of view, the candidate in the $i$-th position can be seen as the preimage of $i$ by $r$, i.e. $r_{i}=r^{-1}(i)$.

${ }^{4}$ These conventions are aimed at simplifying the proofs and do not interfere with the search of optimal rankings since $a_{\emptyset}$ is not in $C$.
} 


\subsection{Unavailable Candidate Model}

We consider a setting where the availability of the candidates is uncertain. We assume a probability distribution $P$ on $\mathscr{P}(C)$ that we will refer to as the unavailability distribution (not necessarily known by the voters). For every $S \subseteq C$, $P(S)$ denotes the probability that the set of available candidates is exactly $S^{5}$. We use $\mathcal{P}_{C}$ to denote the set of probability distributions on $\mathscr{P}(C)$. Given $P \in \mathcal{P}_{C}$ and an application $g$ defined on $\mathscr{P}(C)$, the expectation of $g$ under $P$ is $\underset{S \sim P}{\mathbb{E}_{P}}[g(S)]:=\sum_{S \subseteq C} P(S) g(S)$.

Definition 1. $P \in \mathcal{P}_{C}$ is pair-sensitive if $\forall S \subseteq C,|S|=2 \Rightarrow P(S)>0$.

Definition 2. $P \in \mathcal{P}_{C}$ is said impartial if it satisfies $\forall S \subseteq C, \forall S^{\prime} \subseteq C,|S|=$ $\left|S^{\prime}\right| \Rightarrow P(S)=P\left(S^{\prime}\right)$. $\tilde{\mathscr{P}}_{C}$ denotes the set of impartial probability distributions of $\mathcal{P}_{C}$. We then define, for $P \in \tilde{\mathscr{P}}_{C}, \tilde{P}$ as follows: for $k \in \llbracket 1 ; m \rrbracket, \tilde{P}(k)$ is the probability $P(S)$ for any $S \subseteq C$ of cardinality $k^{6}$. $P$ is impartial means that $P$ treats all the candidates equally.

$\mathrm{Lu}$ and Boutilier [15] propose to evaluate a ranking with respect to the expected disagreement that measures, for each voter, the binary disagreement that evaluates to one if the winner (the elected candidate) is not the same as the voter's preferred choice among the available choices and zero otherwise. Assuming that the probability of a candidate to be unavailable is $p \in] 0 ; 1[$, the expected number of disagreements between two rankings $r$ and $r^{\prime}$ is defined as

$$
D_{p}\left(r, r^{\prime}\right):=\sum_{S \subseteq C} p^{m-|S|}(1-p)^{|S|} \mathbb{1}\left[\operatorname{top}_{r}(S) \neq \operatorname{top}_{r^{\prime}}(S)\right]
$$

Observation 1 D $D_{p}$ is a metric.

An optimal ranking is a ranking that minimises $\sum_{v \in V} D_{p}(r, v)$. Note that $D_{p}$ implicitly assumes that the best decision would be to follow plurality when the set of available candidates is revealed. Since a ranking has to be produced before the set $\mathrm{S}$ is revealed, the optimal ranking is the one that best approximates "plurality a posteriori". Plurality is a rule that is not perceived satisfactory from the point of view of social theory [14] because it only takes into account the first candidate of the voter's preference and thus loses a lot of information. Precisely, a major advantage of plurality is its simplicity and the small quantity of information needed and, thus, the cognitive load for the voters is reduced. This is not the case in the UCM where the complete ranking of preferences is anyway needed for each voter. Generalising scoring rules via ex post dissatisfaction and not only plurality provides a richer model and does not require more information, except the disutility functions which may be, in a lot of scenarios, the same for all voters.

\footnotetext{
${ }^{5}$ The atomic elements being the subsets of $C, P$ should actually be defined on $\mathscr{P}(\mathscr{P}(C))$ and the probability that the set of available candidates is equal to $S$ would be $P(\{S\})$. We nevertheless write $P(S)$ for the sake of readability.

${ }^{6}$ The notation $\tilde{P}(k)$ must not be confused with the probability that the set of available candidates is of cardinality $k$, which is actually equal to $\tilde{P}(k) \times\left(\begin{array}{c}m \\ k\end{array}\right)$.
} 


\section{Generalised unavailable candidate model}

In this work we provide a generalisation of the UCM by evaluating a ranking with respect to the expected dissatisfaction that it imposes on the voters. A ranking defines a policy for making a choice under uncertain availability of candidates. The final choice is the top ranked candidate among the available ones. The voters suffer a degree of dissatisfaction that depends on the position of the final choice in their own ranking. In order to introduce our model, as the first thing, we need to define a function that maps rank positions to "disutility" values.

Definition 3. $A$ disutility function $(D F) \rho$ is an increasing mapping from $\llbracket 1 ; m \rrbracket$ to $\mathbb{R}$. $\rho$ may be represented as a sequence i.e. $\rho=(\rho(i))_{i \in \llbracket 1 ; m \rrbracket} . \rho(i)$ measures how much a voter is unsatisfied by the item at the $i$-th position in its ranking.

The overall idea of our generalised model is the following. Given a ranking $r$, when the set $S$ of available candidates is observed, the candidate $\operatorname{top}_{r}(S)$ is declared the winner; in other words the winner is the highest ranked candidate in $S$ with respect to $r$. As far as the voter $v$ is concerned, its most desired candidate is $\operatorname{top}_{v}(S)$. We allow the disutility functions to depend on the voter and note $\rho_{v}$ the disutility function associated to voter $v$. This implies that, in the generalised UCM, considering a voter $v$ is actually considering the ranking of the preference of $v$ and the $\mathrm{DF} \rho_{v}$, the definition of $\mathscr{V}$ being consequently adapted. Nevertheless, in the absence of ambiguity and to lighten the notations, $v$ may refer to either the voter or its preference order in the remainder of the paper. $\rho_{v}$ and the position in $v$ of a candidate $a$ determine the disutility that voter $v$ suffers from the election of $a$. The dissatisfaction, with respect to $S$, is then the difference between the disutility of $\operatorname{top}_{r}(S)$ and that of $\operatorname{top}_{v}(S)$. Finally, the total dissatisfaction is the sum of the dissatisfactions of all the voters in $V$. When we produce a ranking, the set of available candidates is not known but we know the distribution $P$. We thus aim at providing the ranking that minimises the total dissatisfaction in expectation over $P$.

In the following we make this reasoning more concrete and discuss two different methods to compute dissatisfaction that differ on whether full or restricted rankings are considered.

\subsection{Ex ante dissatisfaction}

In this approach, the dissatisfaction is computed using the positions of the candidates in the whole set of candidates $C$. When a candidate $a \in S$ is chosen as winner, with $S$ being the set of available candidates, voter $v$ suffers disutility $\rho_{v}(v(a))$, i.e. the disutility associated with the position of a in the full ranking $v$, while its most preferred candidate in $S$ would have given him disutility $\rho_{v}\left(v\left(\operatorname{top}_{v}(S)\right)\right)$; this gives $\rho_{v}(v(a))-\rho_{v}\left(v\left(\operatorname{top}_{v}(S)\right)\right)$ as value of dissatisfaction. We then take the expectation of such a value under $P$, since the set $S$ is not known beforehand. Finally, we obtain $\hat{\Delta}_{P}(v, r)$, that we formally define now. 
Definition 4. Let $P \in \mathcal{P}_{C}$ be a probability distribution, $v$ be a voter (or ranking), $\rho_{v}$ be the DF associated to $v$, and $r$ a ranking. We define

$$
\hat{\Delta}_{P}(v, r):=\underset{S \sim P}{\mathbb{E}}\left[\rho_{v}\left(v\left(\operatorname{top}_{r}(S)\right)\right)-\rho_{v}\left(v\left(\operatorname{top}_{v}(S)\right)\right)\right]
$$

and, if $V \in \mathscr{V}, \hat{\Delta}_{P}(V, r):=\sum_{v^{\prime} \in V} \hat{\Delta}_{P}\left(v^{\prime}, r\right)$.

Proposition 1. For any $P \in \mathcal{P}_{C}, \hat{\Delta}_{P} \geq 0$ and, for any voter $v, \hat{\Delta}_{P}(v, v)=0^{7}$.

We call $\hat{\Delta}_{P}$ the ex ante dissatisfaction measure associated to $P$ and $\rho_{v}\left(v\left(\operatorname{top}_{r}(S)\right)\right)-\rho_{v}\left(v\left(\operatorname{top}_{v}(S)\right)\right)$ is the ex ante dissatisfaction induced by $r \in R$ in $S \subseteq C$ to the voter $v$. The ex ante dissatisfaction induced by $r$ in the empty set to $v$ is $\rho\left(v\left(a_{\emptyset}\right)\right)-\rho\left(v\left(a_{\emptyset}\right)\right)=0$ so the empty set does not contribute to the ex ante dissatisfaction measure. Note that the singletons do not contribute either since they cannot generate dissatisfaction. We also call the application $\hat{\Delta}_{P}$ defined on $\mathscr{V} \times R$, the ex ante dissatisfaction measure associated to $P$.

Definition 5. The optimal rankings for $V \in \mathscr{V}$ are defined as the elements of $\arg \min \left(\hat{\Delta}_{P}(V, r)\right)$. We also write this set $\hat{R}_{P}^{*}(V)$.

$r \in R$

Example 1. Let $m=3, C=\{a, b, c\}$. Let $P \in \mathcal{P}_{C}$ be the uniform probability distribution: for all $S \subseteq C, P(S)=\frac{1}{8}$. Let $n=11$. Let $V \in \mathscr{V}$ be a set of 11 voters, 4 of them voting according to the ranking $r^{\prime}=(a, b, c)$ and 7 of them voting according to the ranking $r^{\prime \prime}=(c, a, b)$. We assume that all voters have the same $\mathrm{DF} \rho=(0,1,2)$. Note that, for every $r \in R, \hat{\Delta}_{P}(V, r)=\underset{S \sim P}{\mathbb{E}}\left[4 \rho\left(r^{\prime}\left(\operatorname{top}_{r}(S)\right)\right)+\right.$ $\left.7 \rho\left(r^{\prime \prime}\left(\operatorname{top}_{r}(S)\right)\right)\right]-\chi(V)$ where $\chi(V):=\underset{S \sim P}{\mathbb{E}}\left[4 \rho\left(r^{\prime}\left(\operatorname{top}_{r^{\prime}}(S)\right)\right)+7 \rho\left(r^{\prime \prime}\left(\operatorname{top}_{r^{\prime \prime}}(S)\right)\right)\right]$ does not depend on $r$. The following array displays, for all $r \in R$, from left to right, the value of $\operatorname{top}_{r}(S)$ for every non-empty and non-singleton $S \subseteq C$, $\sum_{S \subseteq C} \rho\left(r^{\prime}\left(\operatorname{top}_{r}(S)\right), \sum_{S \subseteq C} \rho\left(r^{\prime \prime}\left(\operatorname{top}_{r}(S)\right)\right.\right.$ and $\Sigma=4 \sum_{S \subseteq C} \rho\left(r^{\prime}\left(\operatorname{top}_{r}(\overline{S)})+\right.\right.$ $7 \sum_{S \subseteq C} \rho\left(r^{\prime \prime}\left(\operatorname{top}_{r}(S)\right)=8\left[\hat{\Delta}_{P}(V, r)+\chi(V)\right]\right.$.

\begin{tabular}{c||c|c|c|c||c|c||c}
$\searrow$ & $a b c$ & $a b$ & $a c$ & $b c$ & $\rho\left(r^{\prime}\left(\operatorname{top}_{r}(S)\right)\right.$ & $\rho\left(r^{\prime \prime}\left(\operatorname{top}_{r}(S)\right)\right.$ & $\Sigma$ \\
\hline$(a, b, c)$ & $a$ & $a$ & $a$ & $b$ & $0+0+0+1$ & $1+1+1+2$ & 39 \\
$(a, c, b)$ & $a$ & $a$ & $a$ & $c$ & $0+0+0+2$ & $1+1+1+0$ & $\mathbf{2 9}$ \\
$(b, a, c)$ & $b$ & $b$ & $a$ & $b$ & $1+1+0+1$ & $2+2+1+2$ & 61 \\
$(b, c, a)$ & $b$ & $b$ & $c$ & $b$ & $1+1+2+1$ & $2+2+0+2$ & 62 \\
$(c, a, b)$ & $c$ & $a$ & $c$ & $c$ & $2+0+2+2$ & $0+1+0+0$ & 31 \\
$(c, b, a)$ & $c$ & $b$ & $c$ & $c$ & $2+1+2+2$ & $0+2+0+0$ & 42
\end{tabular}

We deduce that the only optimal ranking is $(a, c, b)$.

\subsection{Ex post dissatisfaction}

In this model, we assume that the disutility felt by a voter when a candidate is elected depends on its position within the set $S$ of the actually available candidates. More precisely, when a candidate $a \in S$ is chosen as winner, voter

\footnotetext{
${ }^{7}$ Referring to the definition of $\hat{\Delta}_{P}$, one can note that the first argument of $\hat{\Delta}_{P}$ here
} is voter $v$ itself while the second argument is its preference order. 
$v$ suffers disutility $\rho_{v}\left(v_{S}(a)\right)$, that is the disutility value associated to $v_{S}(a)$, the position of a in the ranking obtained by restricting $v$ to the set $S$. Voter $v$ 's most preferred candidate in $S$ would have given him disutility $\rho_{v}\left(v_{S}\left(\operatorname{top}_{v}(S)\right)\right)=$ $\rho_{v}(1)$, yielding dissatisfaction $\rho_{v}\left(v_{S}(a)\right)-\rho_{v}(1)$. The expectation of such value under $P$ gives $\Delta_{P}(v, r)$, that we formally define now.

Definition 6. Let $P \in \mathcal{P}_{C}, v$ be a voter (or ranking) with $D F \rho_{v}$ and $r$ be a ranking.

$$
\Delta_{P}(v, r):=\underset{S \sim P}{\mathbb{E}}\left[\rho_{v}\left(v_{S}\left(\operatorname{top}_{r}(S)\right)\right)-\rho_{v}(1)\right] .
$$

and, if $V \in \mathscr{V}, \Delta_{P}(V, r):=\sum_{v^{\prime} \in V} \Delta_{P}\left(v^{\prime}, r\right)$.

Proposition 2. For any $P \in \mathcal{P}_{C}, \Delta_{P} \geq 0$ and, for any voter $v, \Delta_{P}(v, v)=0$.

We call $\Delta_{P}$ the ex post dissatisfaction measure associated to $P$ whether it is defined on $R^{2}$ or $\mathscr{V} \times R$. For a voter $v$, a ranking $r, S \subseteq C, \rho_{v}\left(v_{S}\left(\operatorname{top}_{r}(S)\right)\right)-\rho_{v}(1)$ is the ex post dissatisfaction induced by $r$ in $S$ to $v$. The ex post dissatisfaction induced by $r$ in the empty set to $v$ is $\rho_{v}\left(v_{\emptyset}\left(a_{\emptyset}\right)\right)-\rho_{v}(1)=\rho_{v}(1)-\rho_{v}(1)=0$ so the empty set does not contribute to the ex post dissatisfaction measure. As in the ex ante approach, the singletons do not contribute either.

Definition 7. The optimal rankings for $V \in \mathscr{V}$ are defined as the elements of $\underset{r \in R}{\arg \min }\left(\Delta_{P}(V, r)\right)$. We also write this set $R_{P}^{*}(V)$.

Example 2. We will take the situation of Example 1 and study it with the ex post approach. We keep the same notations as in Example 1. Let $r \in R$. Since $\rho(1)=0$, we directly have $\left.\Delta_{P}(V, r)=\underset{S \sim P}{\mathbb{E}}\left[4 \rho\left(r_{S}^{\prime}\left(\operatorname{top}_{r}(S)\right)\right)\right]+7 \rho\left(r_{S}^{\prime \prime}\left(\operatorname{top}_{r}(S)\right)\right)\right]$. Let us summarise the computations in the following array, as in Example 1:

\begin{tabular}{c||c|c|c|c||c|c||c}
$r$ & $a b c$ & $a b$ & $a c$ & $b c$ & $\rho\left(r_{S}^{\prime}\left(\operatorname{top}_{r}(S)\right)\right.$ & $\rho\left(r_{S}^{\prime \prime}\left(\operatorname{top}_{r}(S)\right)\right.$ & $\Sigma$ \\
\hline$(a, b, c)$ & $a$ & $a$ & $a$ & $b$ & $0+0+0+0$ & $1+0+1+1$ & 21 \\
$(a, c, b)$ & $a$ & $a$ & $a$ & $c$ & $0+0+0+1$ & $1+0+1+0$ & 18 \\
$(b, a, c)$ & $b$ & $b$ & $a$ & $b$ & $1+1+0+0$ & $2+1+1+1$ & 43 \\
$(b, c, a)$ & $b$ & $b$ & $c$ & $b$ & $1+1+1+0$ & $2+1+0+1$ & 40 \\
$(c, a, b)$ & $c$ & $a$ & $c$ & $c$ & $2+0+1+1$ & $0+0+0+0$ & $\mathbf{1 6}$ \\
$(c, b, a)$ & $c$ & $b$ & $c$ & $c$ & $2+1+1+1$ & $0+1+0+0$ & 27
\end{tabular}

Hence, the optimal ranking is $(c, a, b)$. Note that this is a different ranking from the optimal ranking obtained in the ex ante approach, namely $(a, c, b)$.

The question whether the ex ante or the ex post approach is more relevant is open. On the one hand, the ex ante model captures the idea that the "utility" perceived by a voter when a candidate is elected should be independent of whether another candidate is available or not. On the other hand, the ex post approach is more relevant if we want to focus on the regret of the voters: if the candidate $a$ is elected, the more available candidates that voter $v$ preferred to $a$ have been excluded, the more regret $v$ will experiment. We also believe that the ex post approach reduces the incentive of manipulating with regards to the unavailability distribution. Indeed, a voter who knows the unavailability distribution (but not the preferences of the other voters) may use this knowledge to 
manipulate the vote in both approaches. Nevertheless, in a situation where two candidates are much more likely to be available than the others for instance (case of a partial unavailability distribution), the benefit of manipulating must be higher in the ex ante approach since ranking these two candidates at the extreme positions (first and last) will increase the influence of the voter in the result of the aggregation. Quantitative results confirming this intuition would require further work. We will see in the following that both ex ante and ex post models are interestingly linked to several well known voting rules. Note that the ex post approach is also considered in $[2,11]$ and, in particular, by $\mathrm{Lu}$ and Boutilier in [15] as the following proposition shows.

Proposition 3. Let $p \in] 0 ; 1\left[. D_{p}\right.$ is the ex post dissatisfaction measure $\Delta_{P}$ whose probability distribution is $P: S \subseteq C \mapsto p^{m-|S|}(1-p)^{|S|}$ (a Bernoulli distribution as defined in Section 5) and with the $D F 1-\mathbb{1}_{\{1\}}=(0,1, \ldots, 1)$ for all voters. We call $1-\mathbb{1}_{\{1\}}$ the binary DF.

Note that Lumet et al. [16] also proposed a double ex ante/ex post approach in a problem of fair allocation of indivisible goods with the assumption that some goods may turn out to be in bad condition and thus unusable. Choosing between their two approaches consists of choosing whether the aggregation over the agents is performed before or after the expectation over the conditions of the objects in the computation of what the authors call the collective utility. The utility of an object in good condition is well defined and fixed. By contrast, in our work in which the aggregator over the voters is simply the sum ${ }^{8}$ and commutes with the expectation, the ex ante/ex post distinction is based on the definition of the dissatisfaction and on the fact that the disutility can be computed either before or after knowing the actual set of available candidates.

\section{Ex ante dissatisfaction}

\subsection{Link with scoring rules}

We now develop the theory of ranking with respect to ex ante dissatisfaction measure and show the connection with positional scoring rules.

Definition 8. Let $V \in \mathscr{V}$. We define $\mathrm{s}_{V}: a \in C \mapsto \sum_{v \in V} \rho_{v}(v(a))$.

Definition 9. Let $V \in \mathscr{V}$. We define $R^{\uparrow}(V)$ the set of rankings where the candidates are ranked in the increasing order of $\mathrm{s}_{V}$ (there can be several such rankings if some candidates have equal scores).

The following theorem characterises the ex ante dissatisfaction rule. It shows that, regardless of the unavailability distribution $P$, an optimal ranking can be found by sorting alternatives with respect to their score. Moreover, if $P$ is

\footnotetext{
${ }^{8}$ Studying other aggregators is a perspective that would allow us to give more focus on fairness in the consensus production.
} 
pair-sensitive, the set of all optimal rankings is the exact output of the scoring rule with scoring function $-\rho_{v}$ for voter $v$. This result echoes the one from [20] which characterises scoring rule via minimisation of the positional Spearman semi-metric, with the restrictive assumption of strictly increasing scores.

Theorem 1 Let $P \in \mathcal{P}_{C}$ and $V \in \mathscr{V}$. Then, $R^{\uparrow}(V) \subseteq \hat{R}_{P}^{*}(V)$.

If, besides, $P$ is pair-sensitive, $R^{\uparrow}(V)=\hat{R}_{P}^{*}(V)$.

Sketch of proof. We show that minimising $\Delta_{P}(V, r)$ amounts to minimising $\psi(V, r)=\underset{S \sim P}{\mathbb{E}}\left[\mathrm{s}_{V}\left(\operatorname{top}_{r}(S)\right)\right]$ and that, for any $S \subseteq C, \mathrm{~s}_{V}\left(\operatorname{top}_{r}(S)\right)$ is minimal if $r \in R^{\uparrow}(V)$. In the case where $P$ is pair-sensitive, if $r \notin R^{\uparrow}(V)$ we can find $i \in \llbracket 1 ; m-1 \rrbracket$ such that $\mathrm{s}_{V}\left(r_{i}\right)>\mathrm{s}_{V}\left(r_{i+1}\right)$. Exchanging the positions of $r_{i}$ and $r_{i+1}$ in $r$ strictly reduces the value of $\psi(V, r)$ so $r$ is not optimal. Note that, if $P$ is not pair-sensitive and $P(\{a, b\})=0$, in the case where $r^{*} \in R^{\uparrow}(V)$ with $r_{m-1}^{*}=a$ and $r_{m}^{*}=b$, then $r$ deduced from $r^{*}$ by swapping $a$ and $b$ is also optimal, but not in $R^{\uparrow}(V)$ if $s_{V}(a)<s_{V}(b)$.

Corollary 1. An optimal ranking for $V \in \mathscr{V}$ in the ex ante dissatisfaction model can be found in polynomial time in $m$ and $n$.

Corollary 2. Let $V \in \mathscr{V}$. Let $P \in \mathcal{P}_{C}$ and $P^{\prime} \in \mathcal{P}_{C}$ be two pair-sensitive probability distributions. Then $\hat{R}_{P}^{*}(V)=\hat{R}_{P^{\prime}}^{*}(V)$.

The previous corollary expresses that the set of optimal rankings for $V$ does not depend on the unavailability distribution as long as the latter is pair-sensitive.

Example 3. Let $C=\{a, b, c, d\}$. Let $V$ be the multiset of three voters among which one votes according to $(d, b, a, c)$, one according to $(d, a, b, c)$ and one according to $(b, c, a, d)$. We suppose that all voters have the DF $\rho=(0,1,2,3)$. We have: $\mathrm{s}_{V}(a)=2+1+2=5, \mathrm{~s}_{V}(b)=1+2+0=3, \mathrm{~s}_{V}(c)=3+3+1=7, \mathrm{~s}_{V}(d)=$ $0+0+3=3$. The rankings that rank the candidates in the increasing order of $\mathrm{s}_{V}$ thus are $(b, d, a, c)$ and $(d, b, a, c)$. We deduce that these two rankings are the optimal rankings for $V$ whatever is the pair-sensitive unavailability distribution.

\section{Ex post dissatisfaction}

In this section, we study ex post dissatisfaction rule from an algorithmic point of view and under natural assumptions on the unavailability distribution and the DF. It is obvious that the ex post dissatisfaction is unchanged by a translation on any of the voters' DF. Hence, without loss of generality, we will consider in the following that all the DF are null in 1 and therefore non-negative on $\llbracket 1 ; m \rrbracket$.

Remark 1. Let $P \in \mathcal{P}_{C}$. If all the voters' $\mathrm{DF}$ are null in 1 then, for all $(V, r) \in$ $\mathscr{V} \times R, \Delta_{P}(V, r)=\sum_{v \in V} \underset{S \sim P}{\mathbb{E}} \rho_{v}\left(v_{S}\left(\operatorname{top}_{r}(S)\right)\right)^{9}$.

\footnotetext{
9 This remark motivates our choice of considering disutilities instead of utilities because, when $\rho_{v}(1)=0$ for all $v \in V$, the ex post dissatisfaction measure can be seen as a simple sum of expectations of $\rho_{v}\left(v_{S}\left(\operatorname{top}_{r}(S)\right)\right)$.
} 
Definition 10. A DF $\rho$ is discriminating at the top $(D T)$ if $\rho(1)<\rho(2)$.

Proposition 4. Let $P \in \mathcal{P}_{C}$ pair-sensitive. Let $v$ be a voter with $D F \rho_{v}$. We have $\left(\forall r \in R, \Delta_{P}(v, r)=0 \Longleftrightarrow r=v\right)$ if, and only if, $\rho_{v}$ is DT.

Conversely, let $P \in \mathcal{P}_{C}$ not pair-sensitive. For any voter $v$, there exists a ranking $r \neq v$ such that $\Delta_{P}(v, r)=0$ ( $\rho_{v}$ being either $D T$ or not).

Observation 2 Multiplying all voters' DF by $\alpha \in \mathbb{R}_{+}^{*}$ does not change $R_{P}^{*}(V)$.

Proof. Multiplying all the voters' DF by the same $\alpha$ multiplies $\Delta_{P}$ by $\alpha$.

Definition 11. A DF $\rho$ is overnormalised if it is DT, $\rho(1)=0$ and $\rho(2) \geq 1$.

Proposition 4 incentives us to study only DF that are DT. In this context, Observation 2 shows that we can restrict our study to overnormalised DF without loss of generality - it suffices to multiply all the DF by $\frac{1}{\min _{v \in V} \rho_{v}(2)}$.

As in the work of $\mathrm{Lu}$ and Boutilier [15], we introduce a specific kind of probability distribution of $\mathcal{P}_{C}$. Assuming that the probability of a candidate to be unavailable is independent of the presence of the other candidates and that this probability is equal to $p \in] 0 ; 1$ [ for all candidates, we get the probability distribution $S \subseteq C \mapsto p^{m-|S|}(1-p)^{|S|}$. We call it the Bernoulli distribution of parameter $p$. Note that a Bernoulli distribution is impartial and pair-sensitive.

\subsection{Connections with Kendall's tau metric}

In the following, for any $\left(r, r^{\prime}\right) \in R^{2}, \kappa\left(r, r^{\prime}\right):=\sum_{i<j} \mathbb{1}\left[r_{i}<_{r^{\prime}} r_{j}\right]$ is the Kendall's tau metric between $r$ and $r^{\prime}$ and, for any $(V, r) \in \mathscr{V} \times R, \kappa(V, r):=\sum_{v \in V} \kappa(v, r)$.

Remark 2. Let us suppose that all voters have the DF $\rho=(0,1, \ldots, 1)$, i.e. the binary DF. Let $P$ be the impartial probability distribution of $\mathcal{P}_{C}$ that is nonnull only on the pairs; in other words, for $S \subseteq C$, if $|S|=2, P(S)=\frac{2}{n(n-1)}$, otherwise $P(S)=0$. For any $(V, r) \in \mathscr{V} \times R, \Delta_{P}(V, r)=\frac{2}{n(n-1)} \kappa(V, r)$. Hence, $\Delta_{P}$ is the Kendall's tau metric within a strictly positive factor.

A result of Baldiga and Green in [2] shows that the Kemeny rule and the ex post dissatisfaction rule with binary DF for all voters and general impartial unavailability distribution may produce different rankings and highlights in that way the role of the unavailability distribution.

Definition 12. For $\left(r, r^{\prime}\right) \in R^{2}, i \in \llbracket 1 ; m \rrbracket$, we define $x_{r, r^{\prime}}(i):=\mid\left\{a \in C \mid a>_{r}\right.$ $\left.r_{i}^{\prime} \wedge a<_{r^{\prime}} r_{i}^{\prime}\right\} \mid=r_{S_{i}\left(r^{\prime}\right)}\left(r_{i}^{\prime}\right)-1$ the number of candidates ranked after $r_{i}^{\prime}$ in $r^{\prime}$ but before $r_{i}^{\prime}$ in $r$.

We now recall a proposition established with other notations in the proof of Theorem 11 of [15] that will be useful in the following.

Proposition 5. [15] Let $\left(r, r^{\prime}\right) \in R^{2} . \kappa\left(r, r^{\prime}\right)=\sum_{i=1}^{m} x_{r, r^{\prime}}(i)=\sum_{i=1}^{m-1} x_{r, r^{\prime}}(i)$. 
Definition 13. The Borda DF maps $i \in \llbracket 1 ; m \rrbracket$ to $i-1$.

The Borda DF is overnormalised. The name Borda DF is justified by the fact that, if all voters have the Borda DF and $P \in \mathcal{P}_{C}$ is an arbitrary pairsensitive probability distribution, Theorem 1 shows that the ex ante dissatisfaction rule based on the minimisation of $\hat{\Delta}_{P}$ is equivalent to the Borda rule.

Lemma 1. If $\rho$ is a DF, $P \in \mathcal{P}_{C}$ is a Bernoulli distribution of parameter $p \in$ ] $0 ; 1\left[,\left(r, r^{\prime}\right) \in R^{2}\right.$ and $i \in \llbracket 1 ; m \rrbracket$,

$\sum_{S \in \mathscr{T}_{i}\left(r^{\prime}\right)} P(S) \rho\left(r_{S}\left(\operatorname{top}_{r^{\prime}}(S)\right)\right)=\sum_{j=0}^{x_{r, r^{\prime}}(i)} \rho(j+1)\left(\begin{array}{c}x_{r, r^{\prime}}(i) \\ j\end{array}\right)(1-p)^{j+1} p^{x_{r, r^{\prime}}(i)+i-j-1}$.

The next proposition establishes the link with Kendall's tau.

Proposition 6. Let $p \in] 0 ; 1\left[\right.$ and $P \in \mathcal{P}_{C}$ be the Bernoulli distribution of parameter $p$. Let $r \in R$ and $v$ be a voter with the Borda DF.

It holds: $\Delta_{P}(v, r)=(1-p)^{2} \sum_{i=1}^{m-1} x_{v, r}(i) p^{i-1}$.

Proposition 6 shows that the ex post dissatisfaction measure associated to the Borda DF and a Bernoulli distribution can be seen as a weighted version of the Kendall's tau metric but in a different sense than in [19] or [10] since here the weight associated to an inversion in the computation of $\Delta_{P}(v, r)$ does not depend on the ranks of both candidates but only on the rank of the candidate ranked before in $r$ and after in $v$.

\subsection{Solving the ex post dissatisfaction problem}

Complexity of the ex post dissatisfaction rule In the sequel, given $q \in$ $[1 ;+\infty[$, a DF $\rho$ is said to be $q$-sub-geometrical if $\rho$ is overnormalised and, for any $k \in \llbracket 2 ; m \rrbracket, \rho(k) \leq q^{k-2} \rho(2)$. Theorem 2 shows that optimising ex post dissatisfaction measure is NP-hard.

Theorem 2 We suppose that all the voters have the same DF $\rho$ and that $\rho$ is $q$-sub-geometrical, for a $q \in\left[1 ;+\infty[\right.$. Let $\epsilon \in] 0, \frac{1}{n m(m-1)+1}[$. Let $p \in[\max ((1-$ $\left.\epsilon)^{\frac{1}{m-1}}, \frac{q-(1+\epsilon)^{\frac{1}{m-1}}}{q-1}\right) ; 1\left[{ }^{10}\right.$. Let $P \in \mathcal{P}_{C}$ be the Bernoulli distribution of parameter $p$, and $V \in \mathscr{V}$. Any ranking in $R_{P}^{*}(V)$ is also a Kemeny consensus.

Sketch of proof. Using the assumptions on $p$ and $\rho$, we prove, for all $(V, r) \in$ $\mathscr{V} \times R: \rho(2)(1-p)^{2}(1-\epsilon) \kappa(V, r) \leq \Delta_{P}(V, r) \leq \rho(2)(1-p)^{2}(1+\epsilon) \kappa(V, r)$. The bounds on $\epsilon$ and these inequalities enable us to show a contradiction if we suppose the existence of an optimal ranking which is not a Kemeny consensus.

${ }_{10} \epsilon>0$ so both $(1-\epsilon)^{\frac{1}{m-1}}$ and $\frac{q-(1+\epsilon)^{\frac{1}{m-1}}}{q-1}$ are strictly lower than 1 . 
Corollary 3. For any number of candidates $m \in \mathbb{N}^{*}$, we suppose that the voters' DF are all $q_{m}$-sub-geometrical, with $q_{m} \in\left[1 ;+\infty\left[\right.\right.$. For $m \in \mathbb{N}^{*}$, we consider $\left.\epsilon_{m} \in\right] 0, \frac{1}{n m(m-1)+1}\left[, p_{m} \in\left[\max \left(\left(1-\epsilon_{m}\right)^{\frac{1}{m-1}}, \frac{q_{m}-\left(1+\epsilon_{m}\right)^{\frac{1}{m-1}}}{q_{m}-1}\right) ; 1\left[, P_{m} \in \mathcal{P}_{C}\right.\right.\right.$ the Bernoulli distribution of parameter $p_{m}$, and $V_{m}$ a voting situation for $m$ candidates. The problem of size $m$ of finding $r \in R$ minimising $\Delta_{P_{m}}\left(V_{m}, r\right)$ is NP-hard.

Proof. Since finding a Kemeny consensus is NP-hard [3], Theorem 2 gives us the NP-hardness of the problem where all the voters have the same DF. Therefore, the more general problem where DF depend on the voters is also NP-hard.

A polynomial-time approximation scheme We here exhibit an approximation algorithm for the ex post dissatisfaction rule and present some intermediary results that allow us to prove this is a PTAS under natural assumptions. In this subsection, $P$ denotes the Bernoulli distribution of parameter $p \in] 0 ; 1[$. Firstly, let us introduce some definitions necessary to the construction of the algorithm.

Definition 14. If there is no ambiguity on $P$ and $V$, we define $f:(\mathscr{S}, a) \in$ $\mathscr{P}(\mathscr{P}(C)) \times C \mapsto \sum_{v \in V} \sum_{\substack{S \in \mathscr{S} \\ a \in S}} P(S) \rho_{v}\left(v_{S}(a)\right)$. For $a \in C$, let $f(a):=f(\mathscr{P}(C), a)$.

Observation 3 Let $V \in \mathscr{V}, r \in R . \Delta_{P}(V, r)=\sum_{i=1}^{m} f\left(\mathscr{S}_{i}(r), r_{i}\right)$.

Definition 15. For $V \in \mathscr{V}, r \in R, k \in \llbracket 1 ; m \rrbracket, l \in \llbracket k ; m \rrbracket$, we denote $\Delta_{P}^{k, l}(V, r):=$ $\sum_{v \in V} \sum_{i=k}^{l} \sum_{S \in \mathscr{T}_{i}(r)} P(S) \rho_{v}\left(v_{S}\left(r_{i}\right)\right)=\sum_{i=k}^{l} f\left(\mathscr{S}_{i}(r), r_{i}\right)$ the contribution to $\Delta_{P}(V, r)$ of the subsets of $C$ for which one of the candidates of $\left\{r_{i} \mid i \in \llbracket k ; l \rrbracket\right\}$ is winning according to $r$.

We now define our MyopicTop algorithm (Algorithm 1). It is conceptually similar to the one of $\mathrm{Lu}$ and Boutilier but makes use of a new notion of dominance (Corollary 4) which encapsulates the complexity of ex post dissatisfaction rule and applies to any DF.

Example 4. Let $C=\{a, b, c, d, e\}, p=\frac{1}{4}, K=2, V$ constituted of three voters $(a, b, c, d, e)$, one voter $(a, c, b, d, e)$ and one voter $(c, a, d, e, b)$, all voters with Borda DF. $a$ is dominant in $C$. In $C \backslash\{a\}$, there is no dominant candidate and the rankings of $C \backslash\{a\}$ minimising $\Delta_{P}^{2,3}(V, r)$ are the rankings starting with $(c, b)$. Then, MyopicTop algorithm outputs $(a, c, b, d, e)$ or $(a, c, b, e, d)$.

Lemma 2. Let $V \in \mathscr{V}$ and $a \in C$. If there exists an optimal ranking that ranks $a$ in first position, then, for every candidate $b \in C,(1+p) f(b) \geq(1-p) f(a)$.

Corollary 4. Let $V \in \mathscr{V}$ be a voting situation and $a \in C$. If, for all $b \in C \backslash\{a\}$, $(1+p) f(a)<(1-p) f(b)$, then, $a$ is the first candidate of all optimal rankings. In this case, we call a the dominant candidate.

Proposition 7. The MyopicTop algorithm runs in $O\left(n m^{\max (3, K+2)}\right)$. 


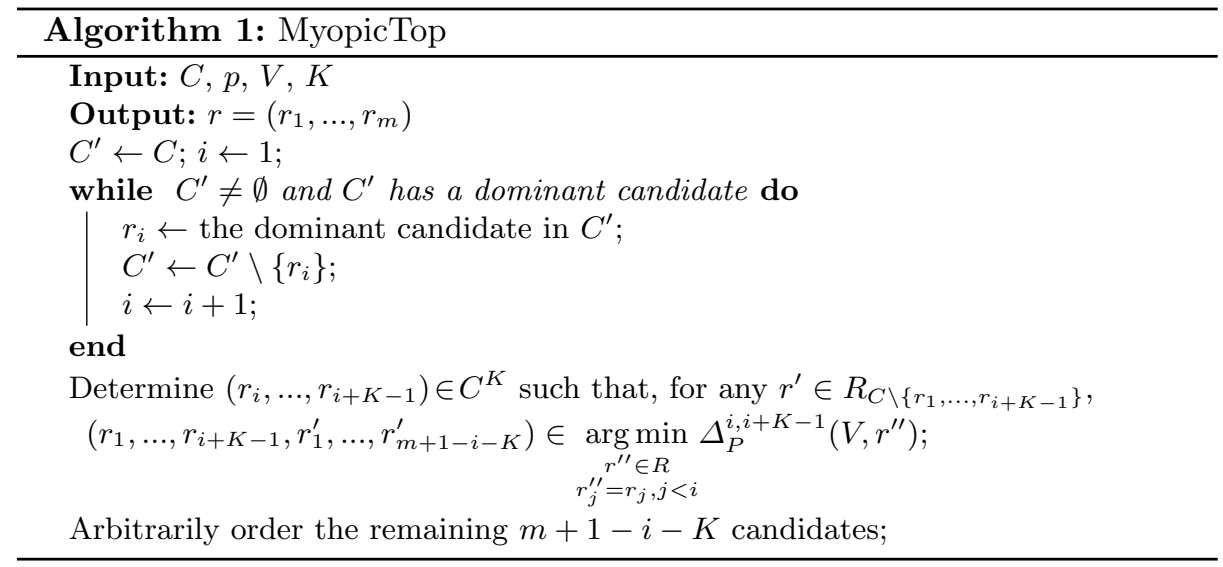

Sketch of proof. Lemma 1 shows that the while loop can be performed in $O\left(\mathrm{~nm}^{3}\right)$. The second part of the algorithm needs to test only $\frac{m !}{(m-K) !}=O\left(m^{K}\right)$ rankings since, once the $i-1$ first candidates are fixed, $\Delta_{P}^{i, i+K-1}(V, r)$ only depends on the candidates between the $i^{\text {th }}$ and the $i+K-1^{t h}$ positions in $r$. Besides, we can reuse Lemma 1 to show that $\Delta_{P}^{i, i+K-1}$ is computed in $O\left(n m^{2}\right)$. Hence, the second part is performed in $O\left(n m^{K+2}\right)$. The last part is done in $O(m)$.

Lemma 3 allows us to apply Corollary 4 to subsets of $C$. We can then show Theorem 3 which proves that MyopicTop algorithm is a PTAS for the ex post dissatisfaction rule when the normalised DF are bounded ${ }^{11}$. Note that here parameter $p$ is assumed to be fixed and independent on $n$ and $m$. This differs from the assumptions made to establish the NP-hardness result in Theorem 2. The question whether the problem with $p$ fixed is NP-hard or not remains open.

Lemma 3. Let $V \in \mathscr{V}, r^{*} \in R_{P}^{*}(V)$ be an optimal ranking. For all $k \in \llbracket 1 ; m \rrbracket$, $\left(r_{k}^{*}, \ldots, r_{m}^{*}\right) \in \underset{r \in R_{S_{k}\left(r^{*}\right)}}{\arg \min }\left(\sum_{v \in V} \sum_{S \in \mathscr{S}_{k}\left(r^{*}\right)} P(S)\left[\rho_{v}\left(v_{S}\left(\operatorname{top}_{r}(S)\right)\right)\right]\right)$ i.e. $\left(r_{k}^{*}, \ldots, r_{m}^{*}\right)$ is an optimal ranking for the reduced set of candidates $S_{k}\left(r^{*}\right)=\left\{r_{k}^{*}, . ., r_{m}^{*}\right\}$.

Theorem 3 We consider voting situations where voters' DF are overnormalised and bounded by a fixed $M \in \mathbb{R}_{+}^{*}:$ for any voter $v$, for all $i \in \llbracket 1 ; m \rrbracket, \rho_{v}(i) \leq M$. Let $\epsilon>0, K=\left\lceil\log _{\frac{1}{p}}\left(\frac{2 M}{(1-p)^{3} \epsilon}\right)\right\rceil$ and $V \in \mathscr{V}$. Let $r^{*} \in R_{P}^{*}(V)$ be an optimal ranking and $r$ be the ranking obtained via the MyopicTop algorithm with inputs $C, p, V, K$. If $\Delta_{P}\left(V, r^{*}\right)=0$ then $\Delta_{P}(V, r)=0$. Otherwise, $\frac{\Delta_{P}(V, r)}{\Delta_{P}\left(V, r^{*}\right)} \leq 1+\epsilon$.

Sketch of proof. First of all, we show that, if the while loop runs until there is no remaining candidates, then $r=r^{*}$. This happens, for instance, when $\Delta_{P}\left(V, r^{*}\right)=0$ and then, in this case, we also have $\Delta_{P}(V, r)=0$. If the while

${ }^{11}$ It is unclear whether there exists a PTAS if the normalised DF are not bounded. 
loop ends before, at an index $i-1$, where $i \in \llbracket 1 ; m \rrbracket, r$ coincides with $r^{*}$ for the first $i-1$ candidates and $\Delta_{P}^{i, i+K-1}(V, r) \leq \Delta_{P}^{i, i+K-1}\left(V, r^{*}\right)$ by construction of $r$. Besides, we show that $\Delta_{P}^{i+K, m}(V, r) \leq n M p^{i+K-1}$ and, the fact that $r_{i}^{*}$ is not dominant in $\mathscr{S}_{i}\left(r^{*}\right)$ enables us to show that $f\left(\mathscr{S}_{i}\left(r^{*}\right), r_{i}^{*}\right) \geq \frac{n}{2} p^{i-1}(1-p)^{3}$. Finally, by construction of $K$, we get $\frac{\Delta_{P}(V, r)}{\Delta_{P}\left(V, r^{*}\right)} \leq 1+\frac{\Delta_{P}^{i+K, m}(V, r)}{f\left(\mathscr{S}_{i}\left(r^{*}\right), r_{i}^{*}\right)} \leq 1+\frac{2 M p^{K}}{(1-p)^{3}} \leq 1+\epsilon$.

Note that, for the algorithm to be polynomial, the bound $M$ must not depend on the number $m$ of candidates. The assumption whereby the DF are bounded may seem restrictive but is actually quite reasonable if we suppose that a voter cannot cognitively conceive an unbounded dissatisfaction. Indeed, one can naturally consider that, after a fixed rank, all alternatives are equally disliked by the voter, the disutility value $M$ would then mean "I completely dislike this alternative". DF can even be strictly increasing, but converging towards $M$. Hence, this upperbound assumption models the increasing difficulty for the voters to discriminate between alternatives as they go further in their preference rankings.

\section{Conclusion}

We provided an extension of the UCM accounting for rank-based dissatisfaction. The voters' preferences are aggregated in a ranking that minimise the overall expected dissatisfaction and is used to select the winner once the available candidates are known. We considered two different settings, ex ante and ex post, corresponding to different ways of defining dissatisfaction, provided a theoretical analysis of both cases, and gave algorithms for finding or approximating an optimal ranking. This analysis showed that the assumption used to define dissatisfaction has a crucial impact on the complexity of the voting rule. Interestingly, we showed that this two-sided model provides a unified representation for very different voting rules, spanning from positional scoring rules to Kemeny rule.

Future works may include analysis of practical performance with simulations. We now provide some theoretical directions of research. First of all, we are interested in studying other probability distributions (including non impartial ones) than Bernoulli and thereby emphasising the fact that ex post model generalises voting rules studied in $[11,2,15]$. Other aggregators than the sum could enable us to include fairness considerations [16]. A quite different idea, inspired from [15], would be to analyse the link between optimal rankings and optimal policies - i.e. choice functions - in the ex post approach. We believe that there is a connection between the comparison ex ante/ex post optimal rankings and the rationalisability of optimal policies; this would echo Theorem 1 from [2] that links the rationalisability of optimal policies to the influence of the unavailability distribution. More quantitavely, we could study the role of the inconsistency (in the sense of [1]) of the optimal policies. Comparing ex ante and ex post approaches in terms of manipulation could also be fruitful as mentioned in Section 3.2. Finally, we are also interested in studying elicitation of preferences in a context of uncertain availability. 


\section{References}

1. Apesteguia, J., Ballester, M.A.: A measure of rationality and welfare. Journal of Political Economy 123(6), 1278-1310 (2015)

2. Baldiga, K.A., Green, J.R.: Assent-maximizing social choice. Social Choice and Welfare 40(2), 439-460 (2013)

3. Bartholdi, J., Tovey, C.A., Trick, M.A.: Voting schemes for which it can be difficult to tell who won the election. Social Choice and welfare 6(2), 157-165 (1989)

4. Berg, S., Lepelley, D.: On probability models in voting theory. Statistica Neerlandica 48(2), 133-146 (1994)

5. Boutilier, C., Lang, J., Oren, J., Palacios, H.: Robust winners and winner determination policies under candidate uncertainty. In: Twenty-Eighth AAAI Conference on Artificial Intelligence. AAAI, Québec (2014)

6. Brandt, F., Conitzer, V., Endriss, U., Lang, J., Procaccia, A.D.: Handbook of computational social choice. Cambridge University Press (2016)

7. Chevaleyre, Y., Lang, J., Maudet, N., Monnot, J., Xia, L.: New candidates welcome! possible winners with respect to the addition of new candidates. Mathematical Social Sciences 64(1), 74-88 (2012)

8. Dutta, B., Jackson, M.O., Le Breton, M.: Strategic candidacy and voting procedures. Econometrica 69(4), 1013-1037 (2001)

9. Dutta, B., Jackson, M.O., Le Breton, M.: Voting by successive elimination and strategic candidacy. Journal of Economic Theory 103(1), 190-218 (2002)

10. García-Lapresta, J.L., Pérez-Román, D.: Consensus measures generated by weighted kemeny distances on weak orders. In: 2010 10th International Conference on Intelligent Systems Design and Applications. pp. 463-468. IEEE Computer Society, Cairo (2010)

11. Gilbert, H., Portoleau, T., Spanjaard, O.: Beyond pairwise comparisons in social choice: A setwise kemeny aggregation problem. In: Thirty-Fourth AAAI Conference on Artificial Intelligence. pp. 1982-1989 (2020)

12. Klamler, C.: A distance measure for choice functions. Social Choice and Welfare 30(3), 419-425 (2008)

13. Lang, J., Maudet, N., Polukarov, M.: New results on equilibria in strategic candidacy. In: International Symposium on Algorithmic Game Theory. pp. 13-25. Springer (2013)

14. Laslier, J.F.: And the loser is... plurality voting. In: Electoral systems, pp. 327351. Springer (2012)

15. Lu, T., Boutilier, C.E.: The unavailable candidate model: a decision-theoretic view of social choice. In: Proceedings of the 11th ACM conference on Electronic commerce. pp. 263-274 (2010)

16. Lumet, C., Bouveret, S., Lemaître, M.: Fair division of indivisible goods under risk (2012)

17. Naamani-Dery, L., Kalech, M., Rokach, L., Shapira, B.: Reducing preference elicitation in group decision making. Expert Systems with Applications 61, 246-261 (2016)

18. Oren, J., Filmus, Y., Boutilier, C.: Efficient vote elicitation under candidate uncertainty. In: IJCAI. pp. 309-316 (2013)

19. Shieh, G.S.: A weighted kendall's tau statistic. Statistics \& probability letters 39(1), 17-24 (1998)

20. Viappiani, P.: Characterization of scoring rules with distances: application to the clustering of rankings. In: Twenty-Fourth International Joint Conference on Artificial Intelligence (2015) 